\title{
Glycolytic and Mitochondrial Metabolism in Pancreatic Islets from MSG-Treated Obese Rats Subjected to Swimming Training
}

Nayara de Carvalho Leite ${ }^{a}$ Thiago Rentz Ferreira ${ }^{a}$ Sarah Ricklia Patricia Cristine Borck ${ }^{a}$ Paulo Cezar de Freitas Mathias ${ }^{b}$ Henriette Rosa de Oliveira Emilio ${ }^{a}$ Sabrina Grassiollia

aDepartment of General Biology, State University of Ponta Grossa, Ponta Grossa, PR 84030-900, Brazil; 'Department of Cell Biology and Genetics, State University of Maringá, Maringá, PR 87020-900, Brazil

\author{
Key Words \\ Obesity $\bullet$ Exercise $\cdot$ Islets $\bullet$ Glucose metabolism
}

\begin{abstract}
Backgrounds/Aims: Obese rats obtained by neonatal monosodium glutamate (MSG) administration present insulin hypersecretion. The metabolic mechanism by which glucose catabolism is coupled to insulin secretion in the pancreatic $\beta$-cells from MSG-treated rats is understood. The purpose of this study was to evaluate glucose metabolism in pancreatic islets from MSG-treated rats subjected to swimming training. Methods: MSG-treated and control (CON) rats swam for 30 minutes (3 times/week) over a period of 10 weeks. Pancreatic islets were isolated and incubated with glucose in the presence of glycolytic or mitochondrial inhibitors. Results: Swimming training attenuated fat pad accumulation, avoiding changes in the plasma levels of lipids, glucose and insulin in MSG-treated rats. Adipocyte and islet hypertrophy observed in MSG-treated rats were attenuated by exercise. Pancreatic islets from MSG-treated obese rats also showed insulin hypersecretion, greater glucose transporter 2 (GLUT2) expression, increased glycolytic flux and reduced mitochondrial complex III activity. Conclusion: Swimming training attenuated islet hypertrophy and normalised GLUT2 expression, contributing to a reduction in the glucose responsiveness of pancreatic islets from MSG-treated rats without altering glycolytic flux. However, physical training increased the activity of mitochondrial complex III in pancreatic islets from MSG-treated rats without a subsequent increase in glucose-induced insulin secretion.
\end{abstract}

Copyright $(2013$ S. Karger AG, Basel

\section{Introduction}

Maintaining glucose homeostasis is a complex process that depends on perfect synchronism between the synthesis, secretion and sensitivity to insulin hormone produced by pancreatic islets. Disease states such as type 2 diabetes mellitus (T2D) and obesity are 
associated with disruptions in glucose homeostasis. During adipose tissue accumulation, progressive reductions in insulin sensitivity occur that are initially compensated by insulin hypersecretion from pancreatic islets. However, compensatory hyperinsulinaemia over long periods leads to a loss in the secretory capacity of $\beta$-cells and promotes their apoptosis $[1$, $2]$. Thus, $\beta$-cell dysfunction marked by declining insulin secretion leads to hyperglycaemia. Insulin secretion released by $\beta$-cells is tightly regulated by an array of stimulatory and inhibitory factors, with glucose playing a central role. Pancreatic $\beta$-cells respond to rising blood glucose by increasing oxidative metabolism, leading to an increased ATP/ADP ratio in the cytoplasm and subsequently leading to an influx of calcium and insulin secretion. The mechanisms of glucose sensing in pancreatic $\beta$-cells involve the coupling of cytoplasmic and mitochondrial processes. Alterations in the coupling between stimulus and secretion in pancreatic islets from obese humans or rodents are directly involved in the development of T2D $[3,4]$.

Physical training simultaneously reduces the tissue accumulation of adipose and enhances insulin responsiveness, resulting in improved glycaemic control. Exercise can likely prevent the progression from the pre-diabetic insulin resistant condition to overt T2D by diminishing peripheral insulin resistance, consequently reducing the work load on the $\beta$-cells in the pancreas [5]. Although a vast amount of research has been conducted to understand the mechanism by which exercise alters the development of obesity and T2D, its effects on glucose-induced insulin secretion by $\beta$-cells is poorly understood. In terms of pancreatic function, experiments in humans and animals have variously demonstrated that exercise improves insulin resistance, increases insulin sensitivity, increases pancreatic $\beta$-cell mass and generally enhances $\beta$-cell function and insulinotropic action, especially in T2D patients [6]. However, the glucose responsiveness of the pancreatic islets obtained from trained rats varies according to the protocol (acute or chronic exercise), glucose concentration and metabolic state (diabetic, obese or lean rats). For example, in normal rats, intense aerobic exercise acutely inhibits insulin secretion, while in T2D, rats' insulin secretion is increased $[7,8]$. Additionally, the effects of exercise training on glycolytic and mitochondrial pathways in $\beta$-cells are not well understood.

Obesity in both humans and in animal models is associated with insulin hypersecretion. Fasting hyperinsulinaemia is still not clearly understood, but in obese rodents, an increased pancreatic $\beta$-cell response to stimulation plays a significant role [9]. Administration of high doses of monosodium glutamate (MSG) during neonatal development induces hypothalamic lesions that provoke neuroendocrine abnormalities and obesity. Hyperinsulinaemia, glucose intolerance, insulin resistance and dyslipidaemia are hallmarks of obesity that are induced by MSG administration $[10,11]$. Thus, MSG-treated obese rodents reproduce the events of the pre-diabetic state similar to those found in obese humans. Pancreatic islets obtained from MSG-treated obese rats also exhibit insulin hypersecretion in the presence of a high glucose stimulus, similar to other models of obesity [12]. Autonomic nervous system (ANS) imbalances also contribute to the altered insulin secretion observed in islets from MSGtreated obese animals [13]. Pancreatic islets from MSG-treated obese mice subjected to swimming training exhibited improved glucose-induced insulin secretion when subjected to high glucose concentrations [14]. However, contrasting results were obtained in islets isolated from MSG-treated obese rats that were also subjected to swimming training [15]. The purpose of this work was to study the metabolic pathways of glucose-induced insulin secretion in pancreatic $\beta$-cells from lean and MSG-treated obese rats subjected to swimming training during growth.

\section{Materials and Methods}

Animals

All animal protocols were approved by the Ethics Committee of the State University of Ponta Grossa (CEUA number 2860). Male neonatal Wistar rats were subcutaneously injected during the first 5 days of life 
with MSG at a dose of $4 \mathrm{mg} / \mathrm{g}$ body weight (bw). Control (CON) animals received equimolar saline solution. Both animal groups were weaned at 21 days old. All animals were housed under controlled conditions in a 12-h light-dark cycle $(07: 00$ to $19: 00 \mathrm{~h})$ at $21 \pm 2{ }^{\circ} \mathrm{C}$. Water and standard rodent chow (Nuvital - Curitiba BR) were supplied ad libitum.

\section{Swimming training}

After weaning at 21 days, CON and MSG-treated obese rats were divided into 2 groups, exercised (EXE) and sedentary (SED) rats. In the first week of the swimming training program, all of the animals were habituated to the water and adapted to the training protocol. This period consisted of 3 sessions of free swimming per week, with the time per session gradually increased from 5 to 15 and then up to $30 \mathrm{~min}$. After one week of adaptation, each training session consisted of 3 30-min swimming sessions per week. Exercise protocols based on swimming are widely used for rats because they are natural swimmers, and the intensity of the exercise was determined by the amount of lead weight attached to the tail. Once a week, each animal was weighed, and a load weight equivalent to $5 \%$ of body mass was attached to the base of tail to ensure that animals were in constant swimming activity. A study by Araujo et al. (2007) adapted the lactate minimum test to swimming in rats. This protocol verified that blood lactate concentrations of $5.5 \mathrm{mM}$ are achieved at loads equivalent to $5 \%$ body weight (bw). Thus, this swimming program can be considered moderate exercise [16]. Swimming took place in a tank (area: $\left.1 \mathrm{~m}^{2}\right)$ filled with tap water $\left(32 \pm 2{ }^{\circ} \mathrm{C}\right)$. The water temperature was maintained at $32 \pm 2{ }^{\circ} \mathrm{C}$ to eliminate cold-induced stress. A water depth greater than $30 \mathrm{~cm}$ prevented the rats from resting at the bottom of the pool. After swimming, the animals were dried with a towel and returned to their cages. Rats performed this swimming exercise over a period of 10 weeks. Six rats from each group were placed in the pool simultaneously at 16:00 h in the afternoon. Two groups, CON-SED and MSG-SED, remained sedentary during the entire experimental protocol.

\section{Food intake}

After weaning, rats from all groups were weighed, and their food intake was determined 3 times per week by weighing the non-ingested chow. Food intake was calculated as the chow consumed divided by the bw of each animal. In this way, the total area under the curve (AUC) of food consumption versus time was calculated.

\section{Obesity and biochemical parameters}

After $12 \mathrm{~h}$ of fasting, the animals of all groups were sacrificed at 90 days of age by decapitation, and their total blood was collected in heparinised tubes. The plasma obtained from the blood was stored at $-20^{\circ} \mathrm{C}$ until glucose, triglyceride, total cholesterol and high density lipid (HDL) levels were measured using commercial kits (Gold Analisa ${ }^{\circledR}$ ). Low density lipids (LDL) were measured using the Friedewald equation: LDL [Total Cholesterol - HDL - (Triglycerides/5)]. We also measured plasma insulin using a radioimmunoassay (RIA) technique using ${ }^{125}$ I-labeled human insulin (PerkinElmer) as the standard and an antibody against rat insulin. Retroperitoneal, epididymal, mesenteric and inguinal fat pads were removed, washed with saline solution and weighed to estimate the level of obesity induced by MSG treatment. The Lee index [bw (g) ${ }^{1 / 3} /$ nasal-anal length (cm)] was calculated as a predictor of obesity in MSG-treated rodents [17].

\section{Insulin Resistance}

Insulin sensitivity was evaluated using the homeostasis model assessment (HOMA-IR) index as a surrogate marker of insulin resistance. The HOMA-IR was obtained using fasting glucose (mM) x fasting insulin $(\mathrm{mU} / \mathrm{L}) / 22.5$ [18].

\section{Pancreatic islet isolation}

Islets were isolated from the rat pancreas as previously described [19], with adaptations. After anaesthesia (xylazine and ketamine; $0.6 \mathrm{mg}+3 \mathrm{mg} / 100 \mathrm{~g}$ bw, respectively), the abdominal wall was cut and opened. A 10-mL Hank's buffered saline solution (HBSS) containing collagenase type V (1.0 mg/mL) was injected into the rat's common bile duct. The pancreas, swollen with the collagenase solution, was quickly excised and incubated in a plastic culture bottle for $15 \mathrm{~min}$ at $37{ }^{\circ} \mathrm{C}$. This suspension was then filtered with a $0.5-\mathrm{mm}$ metal mesh and washed with HBSS containing $0.1 \%$ bovine serum albumin fraction V (BSA) in 5 continuous washes. Islets were collected with the aid of a microscope. At least 6 rats were used to obtain a 
de Carvalho Leite et al.: Effect of Swimming Training on Glucose Metabolism

pool of pancreatic islets for each group of animals. Pools of 4 islets were pre-incubated for $60 \mathrm{~min}$ in $1 \mathrm{~mL}$ of normal Krebs-Ringer solution containing $120 \mathrm{mM} \mathrm{NaCl}, 4.8 \mathrm{mM} \mathrm{KCl}, 2.5 \mathrm{mM} \mathrm{CaCl}{ }_{2}, 1.2 \mathrm{mM} \mathrm{MgCl}, 24 \mathrm{mM}$ $\mathrm{NaHCO}_{3}$ and $5.6 \mathrm{mM}$ glucose. This solution was gassed with $\mathrm{O}_{2} / \mathrm{CO}_{2}(95 / 5 \%)$ to maintain $\mathrm{pH} 7.4$ and was then supplemented with BSA $(0.1 \%)$. After adaptation to a low glucose $(5.6 \mathrm{mM})$ solution, the islets were incubated for a further $60 \mathrm{~min}$ in the presence of different glucose concentrations: 5.6, 8.3 and $16.7 \mathrm{mM}$. In addition, other pools of islets were incubated with $16.7 \mathrm{mM}$ glucose in Krebs-Ringer in the presence or absence of the following inhibitors of glucose metabolism: iodoacetate (1 mM, IAA), which blocks glycolysis by inhibiting glyceraldehyde-3-phosphate dehydrogenase; $\alpha$-cyano-4-hydroxycinnamate (1 mM, $\alpha \mathrm{CHC})$, which inhibits the mitochondrial pyruvate transporter and thus prevents the uptake of pyruvate into the mitochondrion and its metabolism via the Krebs cycle; sodium fluoroacetate ( $2 \mathrm{mM}, \mathrm{SF})$, which blocks the Krebs cycle enzyme aconitase and halts the Krebs cycle before the production of reduced nucleotides or GTP; and rotenone $(1 \mu \mathrm{M}, \mathrm{ROT})$ and antimycin A (50 nM, ANT), inhibitors of mitochondrial complexes I and III [20].

\section{Morphological Parameters}

Rats were sacrificed by rapid decapitation, and samples of the splenic portion (tail) of the whole pancreas were rapidly removed and cleared of fat. The lower left lobule of the liver and inguinal subcutaneous and visceral mesenteric fat pads were also collected. All of the tissues were fixed in ALFAC solution (85

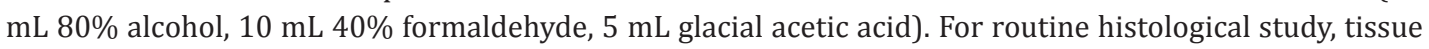
sections were dewaxed with xylene, rehydrated through graded ethanol washes, rinsed in distilled water, stained in haematoxylin and eosin (H\&E), dehydrated through an ascending grade of ethanol solutions and mounted. Three sections $(5 \mu \mathrm{M})$ separated by at least $100 \mu \mathrm{M}$ were examined by light microscopy after H\&E staining. The cell parameters were measured using an image analysis system (Image J 1.39f, NIH - Bethesda, MD, USA). The sectional areas of the adipocytes and islets were determined from digital images acquired at random (Olympus BX51 microscope, LC Evolution camera, TIFF format, 36-bit colour, 1280x1024 pixels); a total of 50 adipocytes were measured per section and all islets were measured in each section. The numbers of adipocytes and islets were also evaluated. Three representative areas were scored in each section, and the average values were used for scoring.

\section{Western blot assay}

Total GLUT2 content was measured in 200-250 isolated pancreatic islets by Western blotting. The protein concentration in the supernatants was assayed using the Bradford assay [21]. The total protein extract levels $(40 \mu \mathrm{g})$ were subjected to SDS-PAGE electrophoresis. For immunoblotting, proteins separated by SDSPAGE were transferred to PVDF membranes. The membranes were blocked for $2 \mathrm{~h}$ at room temperature in a TBS buffer containing 5\% skim milk and then incubated with a primary anti-GLUT2 antibody (1:1000; [cat. (H-67) sc 9117], Santa Cruz, CA, USA). After washing, the membranes were incubated with the secondary antibody (Pierce Biotechnology, IL, USA) and exposed to x-ray film (Amersham Biosciences, Piscataway, NJ) using the ECL Western blotting detection reagent (Amersham Biosciences, Piscataway, NJ). After washing the membranes, $\alpha$-tubulin (1:1000, cat. T6199), Sigma, St. Louis, Mo., USA, detection was used to correct GLUT2 signals for differences in protein loading. The band intensities were quantified by optical densitometry (Scion Image, Frederick, MD).

\section{Chemicals}

Analytical grade reagents and deionised water were used. Collagenase, BSA (fraction V) and the inhibitors ANT, SF, IAA and $\alpha$-CHC were obtained from Sigma (St. Louis, Mo., USA). The inhibitors were dissolved in $100 \%$ dimethyl sulphoxide (DMSO), for a final DMSO concentration of $0.1 \%$ (v/v). The control experiments were performed with the same concentrations of DMSO.

\section{Statistical analysis}

The data are expressed as the mean \pm standard error of the mean (SEM) and compared by an analysis of variance (ANOVA) with a post hoc Bonferroni test. Student's $t$ test was also used. The level of significance was set at $p<0.05$. The statistical analysis was performed using Graph Pad Prism version 5.02 for Windows (Graph Pad Software, San Diego, CA, USA). 


\begin{tabular}{lcccc}
\hline & CON-SED & CON-EXE & MSG-SED & MSG-EXE \\
\hline Body Weight (g) & $333.5 \pm 5.74^{\mathrm{b}, \mathrm{c}, \mathrm{d}}$ & $315.1 \pm 4.29^{\mathrm{a}, \mathrm{c}, \mathrm{d}}$ & $284.7 \pm 5.73^{\mathrm{a}, \mathrm{b}, \mathrm{d}}$ & $264.2 \pm 3.17^{\mathrm{a}, \mathrm{b}, \mathrm{c}}$ \\
Naso-anal Length (cm) & $22.9 \pm 0.13^{\mathrm{c}, \mathrm{d}}$ & $23.0 \pm 0.15^{\mathrm{c}, \mathrm{d}}$ & $20.1 \pm 0.29^{\mathrm{a}, \mathrm{b}}$ & $20.5 \pm 0.27^{\mathrm{a}, \mathrm{b}}$ \\
Lee Index & $0.296 \pm 0.002^{\mathrm{c}}$ & $0.297 \pm 0.002^{\mathrm{c}}$ & $0.303 \pm 0.003^{\mathrm{a}, \mathrm{b}}$ & $0.303 \pm 0.005^{\mathrm{a}, \mathrm{b}}$ \\
Epididymal Fat (g/100g) & $1.20 \pm 0.09^{\mathrm{c}, \mathrm{d}}$ & $1.33 \pm 0.07^{\mathrm{c}, \mathrm{d}}$ & $2.48 \pm 0.11^{\mathrm{a}, \mathrm{b}, \mathrm{d}}$ & $1.94 \pm 0.17^{\mathrm{a}, \mathrm{b}, \mathrm{c}}$ \\
Mesenteric Fat (g/100g) & $0.85 \pm 0.04^{\mathrm{c}, \mathrm{d}}$ & $0.59 \pm 0.02^{\mathrm{c}, \mathrm{d}}$ & $1.69 \pm 0.09^{\mathrm{a}, \mathrm{b}, \mathrm{d}}$ & $1.38 \pm 0.09^{\mathrm{a}, \mathrm{b}, \mathrm{c}}$ \\
Retroperitoneal Fat $(\mathrm{g} / 100 \mathrm{~g})$ & $1.66 \pm 0.16^{\mathrm{c}, \mathrm{d}}$ & $1.51 \pm 0.17^{\mathrm{c}, \mathrm{d}}$ & $3.76 \pm 0.17^{\mathrm{a}, \mathrm{b}, \mathrm{d}}$ & $3.02 \pm 0.19^{\mathrm{a}, \mathrm{b}, \mathrm{c}}$ \\
Inguinal Fat (g/100g) & $0.28 \pm 0.02^{\mathrm{c}}$ & $0.28 \pm 0.02^{\mathrm{c}}$ & $0.77 \pm 0.08^{\mathrm{a}, \mathrm{b}, \mathrm{d}}$ & $0.41 \pm 0.06^{\mathrm{c}}$ \\
\hline
\end{tabular}

Table 1. Biometric parameters in MSG-obese and control rats submitted at swimming training. Data are the mean \pm SEM obtained from $15-20$ rats by group. Letters above of numbers represent statistical difference of $\mathrm{p}<0.05$ in analyses of variance ANOVA with Bonferroni post-test. ${ }^{a}$ CON-SED; ${ }^{b}$ MSG-SED; ${ }^{c} C O N-E X E$ and ${ }^{\mathrm{d}}$ MSG-EXE.

\begin{tabular}{lcccc}
\hline & CON-SED & CON-EXE & MSG-SED & MSG-EXE \\
\hline Basal Insulin (ng/mL) & $0.43 \pm 0.03^{c}$ & $0.31 \pm 0.03^{c}$ & $0.54 \pm 0.05^{\mathrm{a}, \mathrm{b}, \mathrm{d}}$ & $0.41 \pm 0.03^{\mathrm{c}}$ \\
Basal Glucose (mg/dL) & $110.96 \pm 2.40^{c}$ & $109.23 \pm 3.77^{\mathrm{c}}$ & $129.33 \pm 3.12^{\mathrm{a}, \mathrm{b}, \mathrm{d}}$ & $110.89 \pm 3.35^{\mathrm{c}}$ \\
Triglycerides (mg/dL) & $86.56 \pm 7.70^{\mathrm{c}, \mathrm{d}}$ & $89.67 \pm 6.90^{\mathrm{c}, \mathrm{d}}$ & $190.44 \pm 25.51^{\mathrm{a}, \mathrm{b}, \mathrm{d}}$ & $139.52 \pm 7.44^{\mathrm{a}, \mathrm{b}, \mathrm{c}}$ \\
Total Cholesterol (mg/dL) & $87.85 \pm 2.61^{\mathrm{c}}$ & $89.15 \pm 3.63^{\mathrm{c}}$ & $111.10 \pm 4.36^{\mathrm{a}, \mathrm{b}, \mathrm{d}}$ & $94.71 \pm 2.36^{\mathrm{c}}$ \\
HDL (mg/dL) & $44.26 \pm 1.47^{\mathrm{b}, \mathrm{c}}$ & $55.86 \pm 2.82^{\mathrm{a}}$ & $56.10 \pm 4.13^{\mathrm{a}}$ & $53.21 \pm 1.94$ \\
LDL (mg/dL) & $27.38 \pm 1.61^{\mathrm{d}, \mathrm{b}}$ & $17.17 \pm 2.22^{\mathrm{a}}$ & $25.94 \pm 4.48^{\mathrm{c}}$ & $12.40 \pm 3.14^{\mathrm{a}, \mathrm{c}}$ \\
Homa Index & $0.112 \pm 0.01^{\mathrm{c}}$ & $0.091 \pm 0.01$ & $0.183 \pm 0.02^{\mathrm{a}, \mathrm{d}}$ & $0.102 \pm 0.01^{\mathrm{c}}$ \\
\hline
\end{tabular}

Table 2. Blood biochemistry profile in MSG-obese and Control rats submitted at swimming training. Data are the mean \pm SEM obtained from $15-20$ rats by group. Letters above of numbers represent statistical difference of $\mathrm{p}<0.05$ in analysis of variance ANOVA with Bonferroni post-test. a CON-SED; ${ }^{b}$ MSG-SED; ${ }^{c}$ CON-EXE and d MSG-EXE.

\section{Results}

\section{Obesity Model and Swimming Training}

Table 1 reports the effects of neonatal MSG treatment on the different biometric parameters. MSG-SED rats exhibited $15 \%$ and $13 \%$ reductions in bw and naso-anal length, respectively, compared with CON-SED rats. Thus, the Lee Index was 6\% higher in the MSGSED group than in the CON-SED group $(\mathrm{p}<0.05)$. The swimming training had no effect on these parameters for either group. White adipose tissue deposits were approximately 2 -fold higher in MSG-SED rats than in CON-SED rats $(\mathrm{p}<0.05)$. Physical training reduced visceral fat deposits (epididymal, retroperitoneal and mesenteric) by approximately $20 \%$ and reduced subcutaneous adipose tissue deposits (inguinal) by $47 \%$ in the MSG-EXE group compared with the MSG-SED group. However, the MSG-EXE group still had greater adipose tissue content than the CON-SED group $(\mathrm{p}<0.05)$. Swimming training had no effect on white adipose tissue content in CON-EXE.

Table 2 presents the metabolic profile between groups. The plasma levels of glucose and insulin during fasting were $17 \%$ and $25 \%$ higher, respectively, in MSG-SED than in CONSED animals. In addition, the plasma concentrations of the triglycerides and total cholesterol were $118 \%$ and $26 \%$ higher, respectively, in MSG-SED rats compared to CON-SED rats 

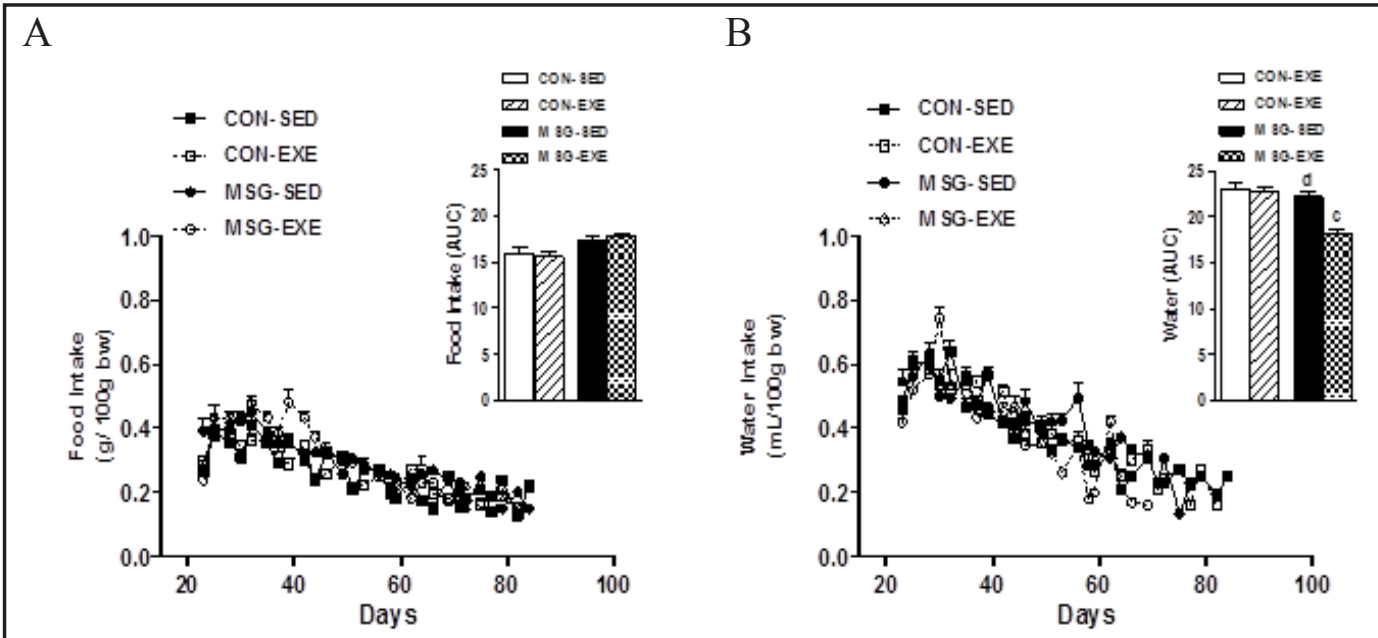

Fig. 1. Food intake and hydric ingestion in MSG-obese and control rats submitted at swimming training. Data are the mean \pm SEM obtained from 15-20 rats by group. The figures $1 \mathrm{~A}$ and $1 \mathrm{~B}$ show consume food intake and water intake evolution. Respective area under curve (AUC) are showed in insert. The symbols in the graphic represent statistical difference of $\mathrm{p}<0.05$ in analyses of variance ANOVA with Bonferroni posttest. ${ }^{a}$ CON-SED; bMSG-SED; ' $\mathrm{C} O N-E X E$ and dMSG-EXE.

$(\mathrm{p}<0.05)$. Swimming training re-established normal glucose, insulin and triglycerides levels in the MSG-EXE group. The plasma LDL levels were similar between the MSG-SED and CONSED groups; however, the HDL levels were higher in the MSG-SED group than in the CONSED group. In both the CON-EXE and MSG-EXE groups, swimming training reduced the LDL concentrations by $39 \%$ and $54 \%$, respectively, compared with the corresponding sedentary groups $(\mathrm{p}<0.05)$. Exercise elevated the HDL levels by $27 \%$ in the CON-EXE group in relation to the CON-SED group $(\mathrm{p}<0.05)$.

Figure 1 demonstrated that neither MSG treatment nor swimming training affected food and water intake in the MSG-SED, CON-SED and CON-EXE groups. Only the MSG-EXE group showed a reduction in water intake compared with the MSG-SED group.

\section{Morphological Parameters}

The maximal adipocyte diameter in the MSG-SED group was significantly higher than that in the CON-SED group. Mesenteric (Fig. 2G) and inguinal adipocyte (Fig. 2C) diameters were $215 \%$ and $110 \%$ greater, respectively, in MSG-SED rats compared to the same deposits in CON-SED rats. There were $77 \%$ and $55 \%$ fewer adipocytes in the mesenteric and inguinal fat pads, respectively, in the MSG-SED group compared with the CON-SED group $(p<0.05)$. In both adipose tissue deposits, physical training reduced the adipocyte diameter by approximately 36\% and 32\% in the CON-EXE (Fig. 2A and 2F) and MSG-EXE (Fig. 2D and 2H) groups, respectively, compared with the corresponding sedentary groups. The swimming training increased the number of adipocytes in both the MSG-EXE and CON-EXE groups by approximately 37\% compared with their sedentary counterparts (Fig. 2B, 2F, 2D and 2H).

Pancreatic islets became hypertrophic in the MSG-SED group in relation to the CON-SED group (Fig. 2K), with 2,400\% larger islet diameters in the MSG-SED group $(\mathrm{p}<0.05)$. Physical training attenuated the islet hypertrophy of the MSG-SED group by approximately $41 \%$ in the MSG-EXE group (Fig. 2L). Neither MSG treatment nor swimming training affected the islet number. 


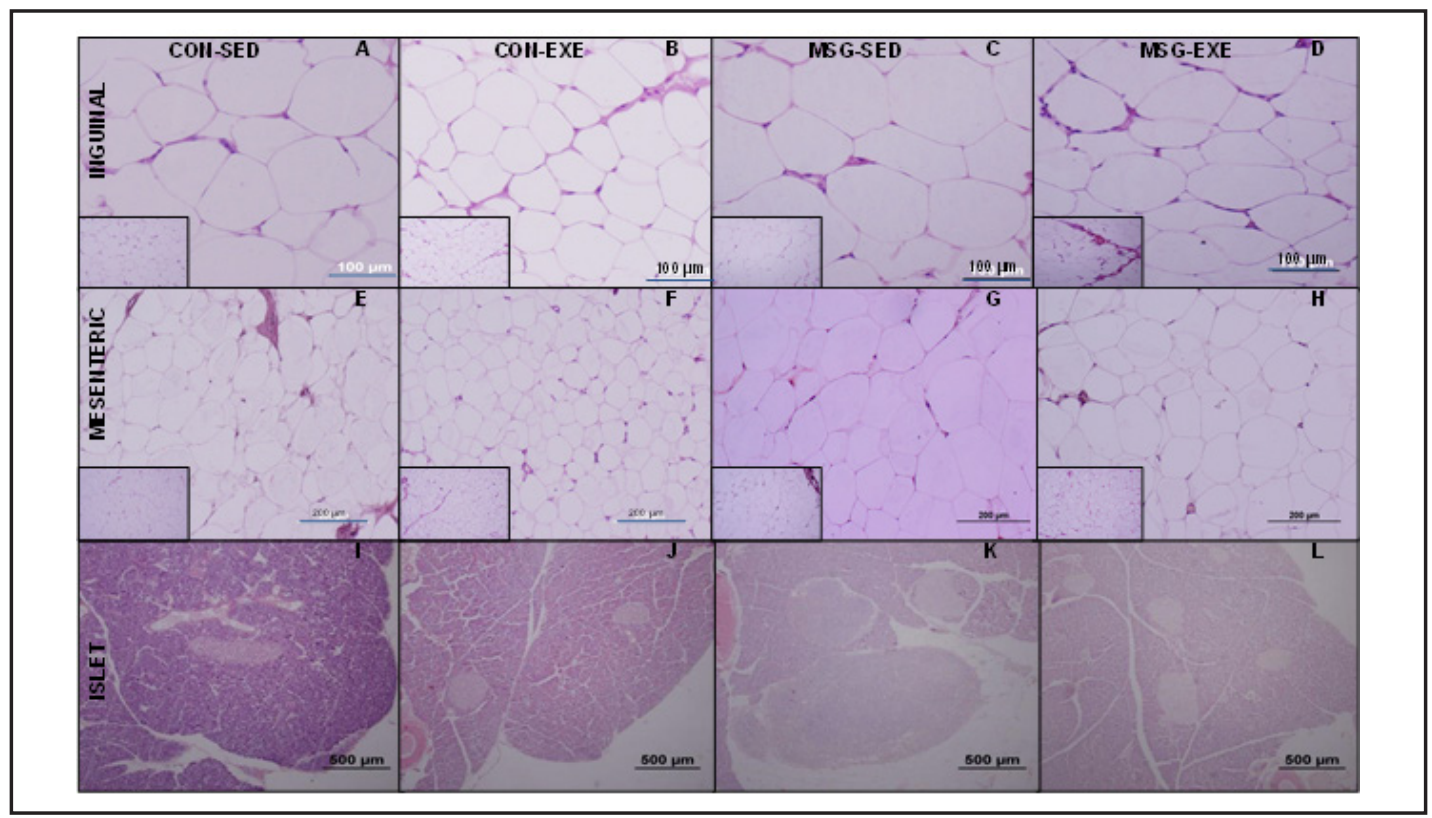

Fig. 2. Effects of swimming training in histomorfological changes in adipose tissue and pancreatic islets from MSG-obese and control rats. Photomicrograph representative of the inguinal adipose tissue (2A-D), mesenteric adipose tissue (2E-H) and pancreatic islets (2I-L). All tissues were stained with hematoxylineosin (H\&E). Micrographs were taken with different objective according indicates in the methods. Morphometric analyses of the area and number were evaluated to adipocytes and islets and respective values are shown as mean \pm SEM in the results.

Fig. 3. Glucose-induced insulin secretion in pancreatic islets from MSG-obese and control rats. Pancreatic islets were isolated by collagenase technique. Groups of 4 islets were incubated in $1 \mathrm{~mL}$ of Krebs solution with different glucose concentration $(5.6 ; 8.3$ and $16.7 \mathrm{mM})$. Values are mean \pm SEM of 20 observations obtained from 4-6 rats by group. Symbols above the bars represent statistical difference of $\mathrm{p}<0.05$ in Student's $t$ test.

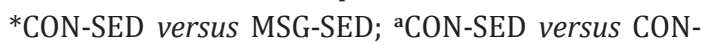
EXE; 'bSG-SED versus MSG-EXE.

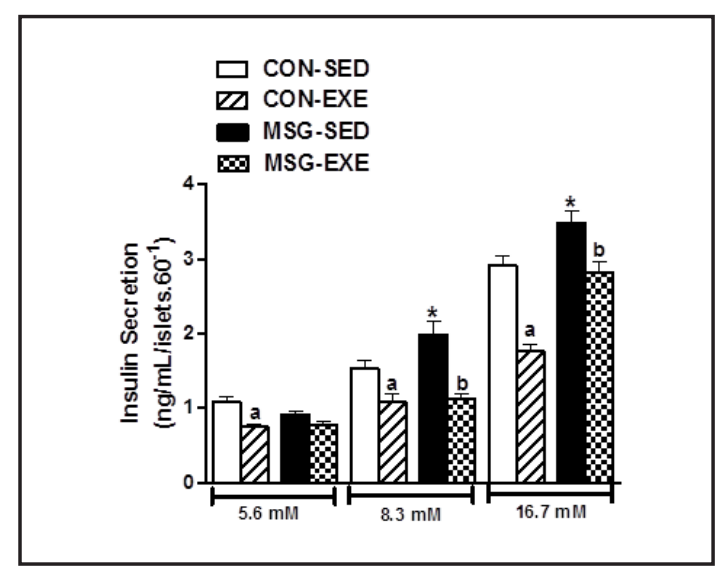

Obesity, Swimming and Isolated Pancreatic Islets

Figure 3 shows the glucose-induced insulin secretion from pancreatic islets. In the presence of 8.3 and $16.7 \mathrm{mM}$ glucose, approximately $25 \%$ more insulin was secreted by islets from MSG-SED rats than by islets from CON-SED rats $(\mathrm{p}<0.05)$. However, under the same conditions (8.3 and $16.7 \mathrm{mM}$ ), glucose-induced insulin secretion was approximately $35 \%$ lower for islets from CON-EXE rats than for islets from CON-SED rats $(\mathrm{p}<0.05)$. Similarly, islets obtained from MSG-EXE rats exhibited 43\% (8.3 mM) and 19\% (16.7 mM) lower glucose-induced insulin secretion than the islets from the MSG-SED group (Fig. 3).

Figure 4 presents the effect of inhibitors of the glycolysis and Krebs cycle on glucoseinduced insulin secretion from pancreatic islets. Isolated pancreatic islets were incubated with a high glucose concentration $(16.7 \mathrm{mM})$ in the presence of several inhibitors of glycolytic metabolism. The blockage of glycolytic flux with IAA reduced glucose-induced 

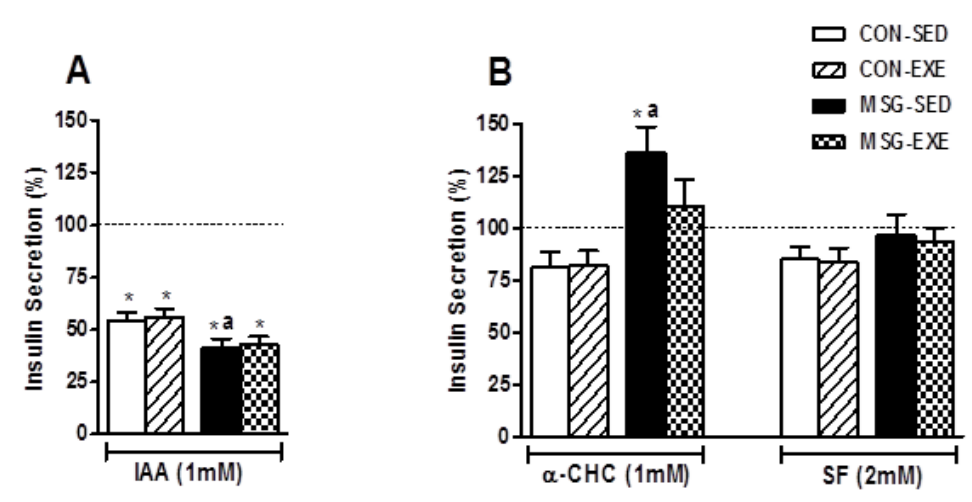

Fig. 4. Effect of swimming training in the glycolysis and Krebs cycle in islets from MSG-obese and control rats. Groups of 4 islets were incubated in $1 \mathrm{~mL}$ of Krebs solution with glucose $(16.7 \mathrm{mM})$ in the presence of inhibitors of glycolysis (left panel) or inhibitors of Krebs Cycle (right panel). Data are showed in relation at $100 \%$ (only glucose $16.7 \mathrm{mM}$ ). Values are mean \pm SEM of 20 observations obtained from $4-6$ rats by groups. Symbols above of bars represent statistical difference of $\mathrm{p}<0.05$ in analysis of variance ANOVA with Bonferroni post-test. ${ }^{*} \mathrm{p}<0.05$ in relation at glucose $16.7 \mathrm{mM}(100 \%)$; ${ }^{\mathrm{C}} \mathrm{CON}-\mathrm{SED}$ versus MSG-SED; ${ }^{\mathrm{b} C O N-S E D}$ versus CON-EXE; 'MSG-SED versus MSG-EXE.

Fig. 5. Effect of swimming training in the mitochondrial metabolism in islets from MSG-obese and control rats. Groups of 4 islets were incubated in $1 \mathrm{~mL}$ of Krebs solution with glucose $(16.7 \mathrm{mM})$ in the presence of inhibitors of complex mitochondrial. Data are showed in relation at $100 \%$ (only glucose $16.7 \mathrm{mM}$ ).Values are mean \pm SEM of 20 observations obtained from 4-6 rats by group. Symbols above of bars represent

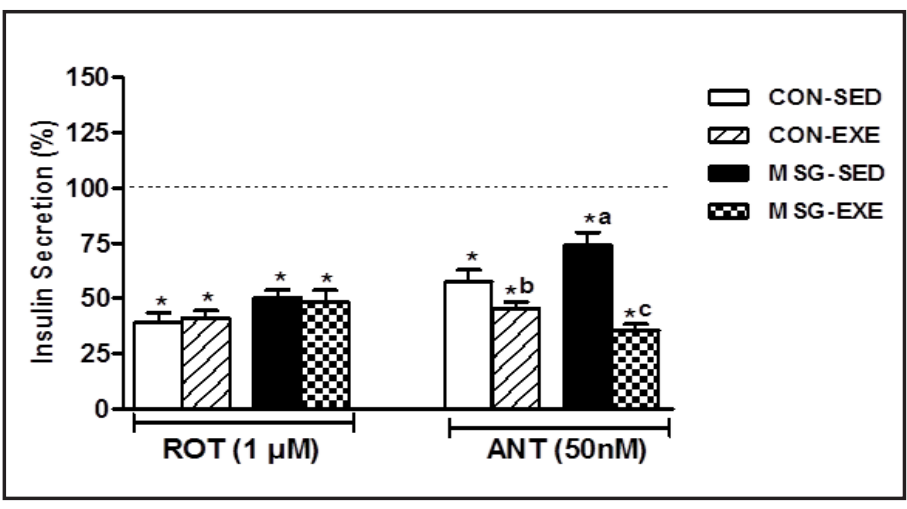
statistical difference of $\mathrm{p}<0.05$ in analysis of variance ANOVA with Bonferroni post-test. ${ }^{*} \mathrm{p}<0.05$ in relation at glucose $16.7 \mathrm{mM}(100 \%)$; ${ }^{a}$ CON-SED versus MSG-SED; ${ }^{b}$ CON-SED versus CON-EXE; ${ }^{c}$ MSG-SED versus MSGEXE.

insulin secretion by pancreatic islets from CON-SED and MSG-SED rats by $46 \%$ and 59\%, respectively (Fig. 4A). The reduction induced by IAA was $44 \%$ and $57 \%$ for islets obtained from the CON-EXE and MSG-EXE groups, respectively (Fig. 4A). However, the inhibitor of the Krebs cycle $(\alpha-\mathrm{CHC})$ had no significant effect on glucose-induced insulin secretion in islets from CON-SED and CON-EXE rats (Fig. 4B). However, glucose-induced insulin secretion in the presence of $\alpha$-CHC was $49 \%$ higher for islets from MSG-SED rats compared with islets from CON-SED rats. This effect was attenuated in islets from MSG-EXE rats compared to islets from MSG-SED rats (Fig. 4B). Independently of swimming training, the addition of SF also had no effect on glucose-induced insulin secretion by islets from CON and MSG-treated obese rats (Fig. 4B).

The effect of mitochondrial inhibitors on glucose-induced insulin secretion from isolated pancreatic islets is presented in Figure 5. The inhibition of mitochondrial complex I with ROT reduced glucose-induced insulin secretion by islets from the CON-SED and MSG-SED groups by $61 \%$ and $50 \%$, respectively. Similar effects were also observed in islets from the exercise groups. ROT reduced glucose-induced insulin secretion by $59 \%$ and $52 \%$, respectively, in islets from CON-EXE and MSG-EXE rats (Fig. 5). The inhibition of mitochondrial complex 
Fig. 6. GLUT2 expression from islets isolated of MSG obese and CON submitted at swimming training. Western blotting analysis of GLUT2 and $\alpha$-tubulin (internal control) expression in protein extracts from islets obtained from MSG obese and CON, sedentary and exercised groups. The bars represent the mean \pm SEM of the values, determined by optical densitometry of 100-150 islets each from three rats of each group $(n=3-$ 6). Symbols above the bars represent statistical difference of $\mathrm{p}<0.05$ in Student's $t$ test. "CON-SED versus MSG-SED; *MSGSED versus MSG-EXE $(\mathrm{p}<0.05)$.

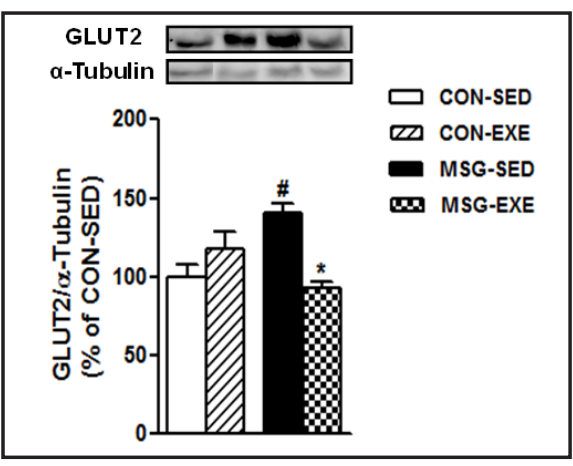

III with ANT also reduced glucose-induced insulin secretion in all groups. However, the magnitude of this response was affected by obesity and exercise. The addition of ANT inhibited glucose-induced insulin secretion by $42 \%$ and $26 \%$ in islets from CON-SED and MSG-SED rats, respectively. In islets from CON-EXE and MSG-EXE rats, ANT reduced glucoseinduced insulin secretion by $55 \%$ and $65 \%$, respectively (Fig. 5).

Glucose transporter 2 (GLUT2) Expression

As illustrated in Figure 6, GLUT2 expression was 41\% higher in islets from MSG-SED rats than in islets from CON-SED rats $(\mathrm{p}<0.05)$. Chronic swimming training reduced GLUT2 content by $48 \%$ in isolated pancreatic islets from MSG-EXE rats when compared with MSGSED rats $(\mathrm{p}<0.05)$.

\section{Discussion}

MSG obesity models and Swimming training

High doses of MSG administered during the neonatal phase damage hypothalamic arcuate nucleus (ARC) inducing the obesity. MSG-treated obese rats exhibit most of the features observed for human obesity, such as excessive accumulation of abdominal adipose tissue, glucose intolerance, insulin resistance, hyperinsulinaemia and dyslipidaemia [22, 23]. The present study confirmed these characteristics. Sedentary lifestyle and high caloric ingestion have been considered the primary causes of obesity in several populations. Regular physical activity is known to be able to revert many of the abnormalities induced by excessive deposition of fat, including T2D [24]. Our work demonstrated that swimming training during growth reduced the accumulation of adipose tissue and avoided alterations in the insulin, glucose and lipid profiles in MSG-treated obese rats. Similar results were obtained previously for MSG-treated obese rats and mice subjected to swimming training ( $1 \mathrm{~h} /$ day; 5 days/week) $[25,7]$. In addition, regular physical training preserves glycaemic homeostasis by restoring insulin sensitivity in peripheral tissues [5]. Our results showed that swimming training maintains fasting insulin levels, avoiding the loss of insulin sensitivity in MSG-treated, exercised rats.

Our protocol of swimming training did not affect food intake in either group. Similar results have been observed in humans subjected to physical training [26]. However, Zucker $(\mathrm{fa} / \mathrm{fa})$ obese rats subjected to swimming training ( $1 \mathrm{~h} /$ day; 5 days/weeks; 4 weeks duration) exhibited exercise-associated anorexia [27]. Important differences can explain these results. While Zucker $(\mathrm{fa} / \mathrm{fa}$ ) obese rats are hyperphagic [28], MSG-treated obese rats are normophagic, as demonstrated by our data.

The Effect of swimming training in the Histological Analyses

Our results also demonstrated that MSG-SED rats have hypertrophic adipocytes. Studies have shown that hypertrophic adipocytes present an altered inflammatory profile directly related to insulin resistance [29]. Additionally, our results also demonstrated the hypertrophic profiles of pancreatic islets in MSG-SED rats. Studies have shown that successful beta cell 
adaptation to insulin resistance includes increasing beta cell mass via hypertrophy and/ or hyperplasia [30]. However, few studies have been conducted to evaluate the endocrine characteristics of the pancreas in MSG-treated obese rats, and the data that have been reported are contradictory $[22,31]$. The proliferation of pancreatic islets is modulated by parasympathetic vagal activity [32]. Vagal hyperactivity is observed in MSG-treated obese rats, and precocious vagotomy has been shown to block hyperinsulinaemia [33]. Thus, we suggested that the islet hypertrophy observed in our data can be attributed to the vagal hyperactivity present in MSG-treated obese rats. For the first time, our study demonstrates that the swimming training program was effective in to attenuate morphological alterations in adipose tissue and pancreatic islets in MSG-treated obese rats. Studies have shown that swimming training restores autonomic imbalance frequently found in MSG-treated mice [13]. We suggest that changes in the autonomic activity induced by chronic swimming training could be involved in these morphological adaptations observed in pancreatic islets and adipocytes from MSG-treated exercise rats.

\section{Insulin Secretion from isolated pancreatic islets and Swimming training}

In addition to insulin resistance, insulin hypersecretion is a hallmark of obesity and T2D. According showed by our data, the pancreatic islets isolated from MSG-treated obese animals exhibited insulin hypersecretion. Similar results were also observed by other authors [22]. The causes of insulin hypersecretion by pancreatic islets from MSG-treated obese rats are unknown. Higher parasympathetic activity is involved in this altered glucose responsiveness of pancreatic islets from MSG-treated obese rats [34]. In addition, insulin hypersecretion observed in islets from MSG-treated rats can be an adaptation to surpass insulin resistance. However, the maintenance of this insulin hypersecretion over time deteriorates the functional secretory capacity of $\beta$-cells and accelerates overt T2D installation [35]. It has been established that physical training restores insulin sensitivity; however, the impact of physical training on the control of insulin secretion from $\beta$-cells remains poorly understood. Our work demonstrated that swimming training leads to a reduction in glucoseinduced insulin secretion in pancreatic islets isolated from both groups. In healthy subjects and lean rats, several studies also have reported the reduction in glucose-induced insulin secretion $[8,36,37]$. Scarce and contradictory data are available regarding the effects of physical training in islets from MSG-treated obese rodents. De Souza et al. 2003 showed that swimming training ( $1 \mathrm{~h} /$ day; 5 days/week; for 10 weeks) had no effect on glucoseinduced insulin secretion by pancreatic islets isolated from MSG-treated obese rats [15]. In contrast, isolated pancreatic islets from MSG-treated obese mice also subjected to swimming training (15 min/day; 3 days/week; for 18 weeks) increased glucose-induced insulin secretion [38]. Considering the autonomic imbalance in MSG-treated obese rats, we believe that the reduction in glucose responsiveness could result from the inhibitory activation of sympathetic nervous system (SNS) to the endocrine pancreas. This hypothesis is reinforced by data showing that swimming training in MSG-treated obese mice increases the neural firing rate of SNS [13].

\section{GLUT2 expression in isolated pancreatic islets}

T2D in rats is associated with a loss of glucose-stimulated insulin secretion and a reduction in the number of GLUT2-positive $\beta$-cells [39]. GLUT2 under-expression has been reported in the $\beta$-cells of Zucker $(f a / f a)$ rats [40] and $\mathrm{db} / \mathrm{db}$ mice, models of spontaneously occurring T2D with antecedent obesity [41]. Significant decreases in both GLUT2 and glucokinase (GK) were also found in rats fed high fat diets (HFD) [42]. Our study demonstrated for the first time that GLUT2 expression is higher in islets from MSG-treated obese rats. This condition could contribute to the high glucose-induced insulin secretion observed in pancreatic islets in this obesity model. We also observed that swimming training promotes the normalisation of GLUT2 expression in MSG-EXE rats. Similar results were obtained for pancreatic islets isolated from diabetic Goto-Kakizaki (GK)-exercised rats (treadmill, 5 days/week, for 9 weeks) [43]. However, increase in GLUT2 expression in pancreatic islets was observed in 
lean trained rat (treadmill; 1, 3 or 5 times per week; for 8 weeks) [44]. In pancreatic islets from the CON-EXE (lean) group, we also observed an increase in GLUT2 expression, but this effect was not significant.

Glucose Metabolism in Isolated Pancreatic islets isolated from swimming trained rats

Glucose-stimulated insulin secretion from the $\beta$-cells of pancreatic islets is a metabolic response that depends critically on cellular ATP. In the presence of glucose, the increase in the intracellular ATP-to-ADP ratio closes the ATP-sensitive $\mathrm{K}^{+}\left(\mathrm{K}^{+}{ }_{\text {ATP }}\right)$ channels, which, in turn, results in the depolarisation of the plasma membrane, the influx of extracellular $\mathrm{Ca}^{2+}$, and the activation of exocytosis [45]. The $\beta$-cells of T2D patients are frequently inadequately responsive to glucose, and the $\beta$-cell mass is frequently reduced [2]. Insulin hypersecretion is also a hallmark of obesity induced by neonatal administration of MSG. Thus, we investigated potential mechanisms for the increased glucose-induced insulin secretion in isolated pancreatic islets from MSG-treated obese rats, as well as the effect of swimming training on glucose metabolism in pancreatic islets from lean and MSG-treated obese rats.

Energetic products arising from glycolytic flux, such as ATP, NADH, and pyruvate, is essential to preserving insulin secretion in pancreatic $\beta$-cells [46]. The glycolytic blocker IAA reduced glucose-induced insulin secretion by islets from all groups, both sedentary and exercised rats. These data reinforce other observations demonstrating that glycolysis is critically involved in glucose-stimulated insulin secretion [47]. However, glycolytic blockers had more impact on glucose-induced insulin secretion by islets from MSG-treated obese rats. These results indicate for the first time that the glycolytic flux is increased in the islets from MSG-treated obese rats and could be contributing to the hypersecretion observed in these islets. Similar data were also observed in isolated pancreatic islets from Zucker $(f a / f a)$ obese rats and ob/ob obese mice [48].

The glycolytic product pyruvate is a substrate for the Krebs cycle in the mitochondria and is suggested to be an important modulator of insulin secretion [49]. However, pyruvatestimulated insulin secretion is not typically observed in pancreatic islet preparations. This observation is the basis of the pyruvate paradox, in which pyruvate oxidation is observed but is not accompanied by insulin secretion [50]. Confirming these literature observations, our study demonstrated that inhibitors of the pyruvate metabolism and Krebs cycle did not affect glucose-induced insulin secretion in isolated pancreatic islets. This result reinforces the primary role of ATP and NADH derived from glycolysis in the glucose-induced insulin secretion from pancreatic islets.

Interestingly, in our study, the blockage of pyruvate transport by $\alpha$-CHC had a mild potentiating effect on glucose-stimulated insulin secretion that was observed only in pancreatic islets isolated from MSG-SED. It appears, thus, that in islets from MSG-treated obese rats pyruvate show alternative metabolism pathway under conditions of impaired mitochondrial pyruvate transport. There are two routes important for the metabolism of glycolysis-derived pyruvate: oxidation by pyruvate dehydrogenase (PDH) to acetyl-CoA or carboxylation by pyruvate carboxylase (PC) to oxaloacetate [50]. Our study did not evaluate PC or PDH activity separately. However, PC and PDH activity were indirectly avoided by the addition of $\alpha$-CHC, which blocks pyruvate transport to the mitochondria. In this situation, pyruvate could be converted to lactate by lactate dehydrogenase (LDH). Studies have shown low LDH activities in pancreatic islets from lean rats [51]. Further studies are needed to reveal the LDH activity, as well as, the role of lactate on glucose-induce insulin secretion in islets from MSG-treated obese rats.

In addition, when the Krebs cycle is blocked, glycolytic NAD(P)H continues to supply electrons to the electron transport chain through the malate-aspartate shuttle and the glycerophosphate shuttle during glucose stimulation [52]. This continuous supply of electrons sustains the elevated [ATP]/[ADP] ratio, which, in turn, continues to stimulate insulin secretion. Thus, the increased glucose-induced insulin secretion in pancreatic islets from MSG-treated obese rats during the inhibition of the pyruvate transport could alternately be explained by the increased activity of shuttles. 
Finally, we demonstrated that neither the glycolytic flux nor the pyruvate metabolism in the Krebs cycle pathways was affected by swimming training in isolated pancreatic islets. These results suggested that the reduced glucose responsiveness found in pancreatic islets isolated from exercised rats is not associated with changes in the glycolytic flux and/or the Krebs cycle. Dissociation between glucose response and metabolic pathways was also observed in other studies of pancreatic islets obtained from exercised rats. For example, pancreatic islets isolated from lean trained rats exhibited an inhibition of glucose-induced insulin secretion and simultaneous increases in anaplerotic and cataplerotic enzymes [8]. Thus, changes in the glucose responsiveness of islets from exercised rats could depend on other factors, such as, neurotransmitters, hormones and/or proliferative adaptations. In support of the latter view, studies of islets from exercised rats demonstrated that endurance training favours $\beta$-cell growth and survival, enhancing antioxidant capacity and reducing reactive oxygen species (ROS) production and apoptotic protein content [8].

The production of ATP in $\beta$-cells is dependent of the cytosolic and mitochondrial reactions. Both, cytosolic NADH and mitochondrial sources of NADH and FADH2 stimulate the mitochondrial electron transport chain. The electron flux establishes a proton gradient across the inner membrane, which provides the energy for ATP synthesis [53]. Mitochondrial metabolism generates substantially more ATP than glycolysis and the production of mitochondrial ATP is critical for glucose-dependent insulin secretion. Using several mitochondrial inhibitors our study also evaluated mitochondrial participation on glucose-induced insulin secretion in MSG-treated obese rats subjected at swimming training. The addition of ROT which specifically inhibits the respiratory chain at complex I inhibited glucose-induced insulin secretion in islets from all groups to a similar degree. However, the addition of ROT did not abolish completely insulin secretion. This observation corroborates with other study showing that the complex I of the electron transport chain does not in itself limit the formation of the proton gradient [54]. In addition, this data also indicates that the participation of complex I of the electron transport chain is intact in islets from MSG-treated rats. Similar results also were obtained in pancreatic islets of GK rats, which are characterised by a deficient insulin response to glucose [55]. In contrast, greater activation of complex I mitochondrial has been demonstrated in pancreatic islets isolated from Zucker $(\mathrm{fa} / \mathrm{fa}$ ) [56]. Again, swimming training had no effect in mitochondrial complex I participation in all groups.

On other hand, our study shows for first time reduced inhibitory effect of ANT on glucoseinduced insulin secretion for pancreatic islets from MSG-treated obese rats indicating that mitochondrial complex III is hypoactive in this obesity model. Contradictory results were observed in islets from $o b / o b$ obese mice. In these islets, the inhibition of mitochondrial metabolism with ANT reduced glucose-stimulated secretion to basal levels in individually perfused pancreatic islets [48]. Mitochondrial alterations were also observed in pancreatic islets isolated from rats subjected to foetal programming [57]. Thus, the hypersecretion of insulin frequently observed in islets of MSG-treated obese rats cannot be attributed at high mitochondrial activation. This suggestion is reinforced by recent data showing that glucose oxidation in response to $16.7 \mathrm{mM}$ glucose was reduced in islets from MSG-treated obese rats [58].

Interestingly, when we evaluated the participation of mitochondrial complex III in isolated pancreatic islets from exercised rats, we observed that the inhibitory effect of ANT on mitochondrial complex III was accentuated by swimming training in both groups. It appears, thus, that swimming training increases the participation of mitochondrial complex III. Singly, this effect should increase ATP production and insulin secretion in pancreatic islets. However, in our results, islets obtained from exercise rats shows reduced glucose-induce insulin secretion. Recently, increased levels of uncoupling protein 2 (UCP2) were observed in isolated pancreatic islets from exercised rats, which could be involved in reduced glucoseinduced insulin secretion [44]. In addition, studies have shown that over-expression of UCP2 in an insulin-producing cell line increase mitochondrial respiration while decreasing the coupling to oxidative phosphorylation; this effect results in a low intracellular ATP level and 
reduced glucose-induced insulin secretion [59]. Taken together, these observations suggest that the greater participation of mitochondrial complex III could be an adaptation of the islets from exercised rats to compensate the proton leakage which occurs through increased UCP2 expression induced by exercise. Finally, in islets from MSG-treated exercise rats these events can improve the participation of mitochondrial complex III normalizing glucoseinduced insulin secretion.

\section{Conclusions}

The damage induced by neonatal MSG administration induces obesity through insulin resistance and insulin hypersecretion. Increased glycolytic flux, hypertrophy of pancreatic islets and higher GLUT2 expression could be involved in the greater glucose responsiveness observed in the islets of MSG-treated obese rats. In addition, mitochondrial complex III is hypoactive in pancreatic islets from MSG-treated obese rats. Chronic swimming training was able in attenuates or reverts histology and secretory abnormalities in islets from MSGtreated obese rats, including corrects mitochondrial complex III activity.

\section{Acknowledgements}

We gratefully acknowledge financial support from the Brazilian Council for Scientific and Technological Development (CNPq).

\section{References}

1 Ma ZA, Zhao Z, Turk J: Mitochondrial dysfunction and $\beta$-cell failure in type 2 diabetes mellitus. Exp Diabetes Res 2012;2012:1-11.

$>2$ Costes S, Langen R, Gurlo T, Matveyenko AV, Butler PC: $\beta$-Cell Failure in Type 2 Diabetes: A Case of Asking Too Much of Too Few? Diabetes 2013;62:327-335.

-3 Seino S, Shibasaki T, Minami K: Dynamics of insulin secretion and the clinical implications for obesity and diabetes. J Clin Invest 2011;121:2118-2125.

4 Buettner C: Is hyperinsulinemia required to develop overeating-induced obesity? Cell Metab 2012;16:691692.

5 Beaudry JL, Riddell MC: Effects of glucocorticoids and exercise on pancreatic $\beta$-cell function and diabetes development. Diabetes Metab Res Rev 2012;28:560-573.

6 de Lemos ET, Oliveira J, Pinheiro JP, Reis F: Regular physical exercise as a strategy to improve antioxidant and anti-inflammatory status: benefits in type 2 diabetes mellitus. Oxid Med Cell Longev 2012;2012:741545.

7 Scomparin DX, Grassiolli S, Gomes RM, Torrezan R, de Oliveira JC, Gravena C, Pêra CC, Mathias PC: LowIntensity swimming training after weaning improves glucose and lipid homeostasis in MSG hypothalamic obese mice. Endocr Res 2011;36:83-90.

-8 Zoppi CC, Calegari VC, Silveira LR, Carneiro EM, Boschero AC: Exercise training enhances rat pancreatic islets anaplerotic enzymes content despite reduced insulin secretion. Eur J Appl Physiol 2011;111:23692374.

-9 Ferrannini E, Ferrannini E, Camastra S, Gastaldelli A, Maria Sironi A, Natali A, Muscelli E, Mingrone G, Mari A: B-cell function in obesity: effects of weight loss. Diabetes 2004:3:S26-33.

10 Olney JW: Brain lesions, obesity, and other disturbances in mice treated with monosodium glutamate. Science 1969;164:719-721.

-11 Hirata AE, Andrade IS, Vaskevicius P, Dolnikoff MS: Monosodium glutamate (MSG)-obese rats develop glucose intolerance and insulin resistance to peripheral glucose uptake. Braz J Med Biol Res 1997;30:671674.

12 Tschöp M, Heiman ML: Rodent obesity models: An overview. Exp Clin Endocrinol Diabetes 2001;109:307319. 
de Carvalho Leite et al.: Effect of Swimming Training on Glucose Metabolism

13 Scomparin DX, Gomes RM, Grassiolli S, Rinaldi W, Martins AG, de Oliveira JC, Gravena C, de Freitas Mathias PC: Autonomic activity and glycemic homeostasis are maintained by precocious and low intensity training exercises in MSG-programmed obese mice. Endocrine 2009;36:510-517.

14 Scomparin DX, Grassiolli S, Marçal AC, Gravena C, Andreazzi AE, Mathias PC: Swim training applied at early age is critical to adrenal medulla catecholamine content and to attenuate monosodium L-glutamate-obesity onset in mice. Life sci 2006;79:2151-2156.

15 De Souza CT, Nunes WM, Gobatto CA, de Mello MA: Insulin secretion in monosodium glutamate (MSG) obese rats submitted to aerobic exercise training. Physiol Chem Phys Med NMR 2003;35:43-53.

16 Araujo GGD, Papoti M, Manchado Fde B, de Mello MA, Gobatto CA: Protocols for hyperlactatemia induction in the lactate minimum test adapted to swimming rats. Comp Biochem Physiol A Biochem Mol Biol 2007;148:888-892.

17 Bernardis LL, Patterson BD: Correlation between 'Lee index' and carcass fat content in weanling and adult female rats with hypothalamic lesions. J Endocrinol 1968;40:527-528.

18 Matthews DR, Hosker JP, Rudenski AS, Naylor BA, Treacher DF, Turner RC: Homeostasis model assessment: insulin resistance and $\beta$-cell function from fasting plasma glucose and insulin concentrations in man. Diabetologia 1985;28:412-419.

19 Lacy PE, Kostianovsky M: Method for the isolation of intact islets of Langerhans from the rat pancreas. Diabetes 1967;16:35-39.

20 Schofl C, Börger J, Lange S, von zur Mühlen A, Brabant G: Energetic Requirement of Carbachol-Induced Ca ${ }^{2+}$ Signaling in Single Mouse B-Cells*. Endocrinology 2000;141:4065-4071.

21 Bradford MM: A rapid and sensitive method for the quantitation of microgram quantities of protein utilizing the principle of protein-dye binding. Anal Biochem 1976;72:248-254.

-22 Morrison JFB, Shehab S, Sheen R, Dhanasekaran S, Shaffiullah M, Mensah-Brown E: Sensory and autonomic nerve changes in the monosodium glutamate-treated rat: a model of type II diabetes. Exp Physiol 2008;93:213-222.

23 Leitner C, Bartness TJ: Food deprivation-induced changes in body fat mobilization after neonatal monosodium glutamate treatment. Am J Physiol Regul Integr Comp Physiol 2008;294:775-783.

24 Golbidi S, Mesdaghinia A, Laher I: Exercise in the metabolic syndrome. Oxid Med Cell Longev 2012;2012:349710.

25 Sagae SC, Grassiolli S, Raineki C, Balbo SL, Marques da Silva AC: Sex differences in brain cholinergic activity in MSG-obese rats submitted to exercise. Can J Physiol Pharmacol 2011;89:845-853.

-26 Martins C, Kulseng B, King NA, Holst JJ, Blundell JE: The Effects of Exercise-Induced Weight Loss on Appetite-Related Peptides and Motivation to Eat. Endocrine Care 2010;95:1609-1616.

27 Kibenge MT, Chan CB: The effects of high-fat diet on exercise-induced changes in metabolic parameters in Zucker fa/fa rats. Metabolism 2002;51:708-715.

28 Chentouf M, Dubois G, Jahannaut C, Castex F, Lajoix AD, Gross R, Peraldi-Roux S: Excessive food intake, obesity and inflammation process in Zucker fa/fa rat pancreatic islets. PLoS One 2011;6:e22954.

29 Alarcon-Aguilar FJ, Almanza-Perez J, Blancas G, Angeles S, Garcia-Macedo R, Roman R, Cruz M: Glycine regulates the production of pro-inflammatory cytokines in lean and monosodium glutamate-obese mice. Eur J Pharmacol 2008;599:152-158.

30 Rutter GA, Parton LE: The $\beta$-cell in type 2 diabetes and in obesity. Front Horm Res 2008;36:118-134.

31 Li PP, Shan S, Chen YT, Ning ZQ Sun SJ, Liu Q Lu XP, Xie MZ, Shen ZF: The PPARalpha/gamma dual agonist chiglitazar improves insulin resistance and dyslipidemia in MSG obese rats. Br J Pharmacol 2006;148:610618.

32 Lausier J, Diaz WC, Roskens V, LaRock K, Herzer K, Fong CG, Latour MG, Peshavaria M, Jetton TL: Vagal control of pancreatic ß-cell proliferation. Am J Physiol Endocrinol Metab 2010;299:E786-793.

-33 Balbo SL, Grassiolli S, Ribeiro RA, Bonfleur ML, Gravena C, Brito Mdo N, Andreazzi AE, Mathias PC, Torrezan R: Fat storage is partially dependent on vagal activity and insulin secretion of hypothalamic obese rat. Endocrine 2007;31:142-148.

-34 de Freitas Mathias PC, Grassiolli S, Rocha DN, Scomparin DX, Gravena C: Transplantation of pancreatic islets from hypothalamic obese rats corrects hyperglycemia of diabetic rats. Transplant Proc 2006;39:193-195.

35 Martins AC, Souza KL, Shio MT, Mathias PC, Lelkes PI, Garcia RM: Adrenal medullary function and expression of catecholamine-synthesizing enzymes in mice with hypothalamic obesity. Life Sciences 2004;74:3211-3222.

-36 Rorsman P, Braun M: Regulation of Insulin Secretion in Human Pancreatic Islets. Annu Rev Physiol 2013;75:1-25. 


\section{Cellular Physiology Cell Physiol Biochem 2013;31:242-256

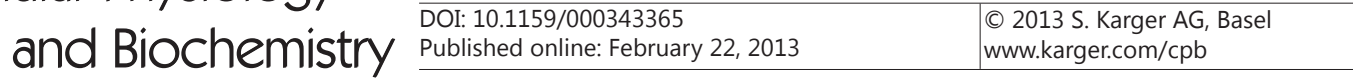 \\ de Carvalho Leite et al.: Effect of Swimming Training on Glucose Metabolism}

-37 Zawalich W, Maturo S, Felig P: Influence of physical training on insulin release and glucose utilization by islet cells and liver glucokinase activity in the rat. Am J Physiol 1982;243:E464-469.

-38 Andreazzi AE, Scomparin DX, Mesquita FP, Balbo SL, Gravena C, De Oliveira JC, Rinaldi W, Garcia RM, Grassiolli S, Mathias PC: Swimming exercise at weaning improves glycemic control and inhibits the onset of monosodium L-glutamate-obesity in mice. J Endocrinol 2009;201:351-359.

-39 Sasaki Y, Suzuki W, Shimada T, Iizuka S, Nakamura S, Nagata M, Fujimoto M, Tsuneyama K, Hokao R, Miyamoto K, Aburada M: Dose dependent development of diabetes mellitus and non-alcoholic steatohepatitis in monosodium glutamate-induced obese mice. Life Sci 2009;85:490-498.

40 Johnson JH, Ogawa A, Chen L, Orci L, Newgard CB, Alam T, Unger RH: Underexpression of $\beta$-cell high Km glucose transporters in noninsulin-dependent diabetes. Science 1990;250:546-549.

41 Mirhashemi F, Kluth O, Scherneck S, Vogel H, Kluge R, Schurmann A, Joost HG, Neschen S: High-Fat, Carbohydrate-Free Diet Markedly Aggravates Obesity but Prevents $\beta$-Cell Loss and Diabetes in the Obese , Diabetes-Susceptible db / db Strain. Obes Facts 2008;1:292-297.

42 Lu H, Koshkin V, Koshkin V, Allister EM, Gyulkhandanyan AV, Wheeler MB: Molecular and Metabolic Evidence for Mitochondrial Defects Associated With B-Cell Dysfunction in a Mouse Model of Type 2 Diabetes. Diabetes 2010;59:448-459.

43 Ueda H, Urano Y, Sakurai T, Kizaki T, Hitomi Y, Ohno H, Izawa T: Enhanced expression of neuronal nitric oxide synthase in islets of exercise-trained rats. Biochem Biophys Res Commun 2003;312:794-800.

-44 Calegari VC, Zoppi CC, Rezende LF, Silveira LR, Carneiro EM, Boschero AC: Endurance training activates AMP-activated protein kinase, increases expression of uncoupling protein 2 and reduces insulin secretion from rat pancreatic islets. J Endocrinol 2011;208:257-264.

45 Henquin JC: Regulation of insulin secretion: a matter of phase control and amplitude modulation. Diabetologia 2009;52:739-751.

46 Chang X, Wei C: Glycolysis and rheumatoid arthritis. Int J Rheum Dis 2011;14:217-222.

-47 Dukes ID, McIntyre MS, Mertz RJ, Philipson LH, Roe MW, Spencer B, Worley JF: Dependence on NADH produced during glycolysis for $\beta$-cell glucose signaling. J Biol Chem 1994;269:10979-10982.

48 Westerlund J, Bergsten P: Glucose Metabolism and Pulsatile Release From Isolated Islets. Diabetes 2001;50:1785-1790.

49 Fridlyand LE, Philipson LH: Glucose sensing in the pancreatic $\beta$-cell: a computational systems analysis. Theor Biol Med Model 2010;15:1-44.

50 MacDonald MJ, Fahien LA, Brown LJ, Hasan NM, Buss JD, Kendrick MA: Perspective: emerging evidence for signaling roles of mitochondrial anaplerotic products in insulin secretion. Am J Physiol Endocrinol Metab 2005;288:E1-15.

51 Ishihara H, Wang H, Drewes LR, Wollheim CB: Overexpression of monocarboxylate transporter and lactate dehydrogenase alters insulin secretory responses to pyruvate and lactate in $\beta$-cells. J Clin Invest 1999;104:1621-1629.

52 Rocheleau JV, Head WS, Nicholson WE, Powers AC, Piston DW: Pancreatic islet $\beta$-cells transiently metabolize pyruvate. J Biol Chem 2002;277:30914-30920.

53 Wollheim CB: Beta-cell mitochondria in the regulation of insulin secretion: a new culprit in type II diabetes. Diabetologia 2000;43:265-277.

54 Antinozzi PA, Ishihara H, Newgard CB, Wollheim CB: Mitochondrial metabolism sets the maximal limit of fuel-stimulated insulin secretion in a model pancreatic $\beta$-cell: a survey of four fuel secretagogues. J Biol Chem 2002;277:11746-11755.

55 Inaba W, Mizukami H, Kamata K, Takahashi K, Tsuboi K, Yagihashi S: Effects of long-term treatment with the dipeptidyl peptidase-4 inhibitor vildagliptin on islet endocrine cells in non-obese type 2 diabetic GotoKakizaki rats. Eur J Pharmacol 2012;691:297-306.

-56 Zhou YP, Cockburn BN, Pugh W, Polonsky KS: Basal insulin hypersecretion in insulin-resistant Zucker diabetic and Zucker fatty rats: role of enhanced fuel metabolism. Metabolism 1999;48:857-864.

57 Theys N, Ahn MT, Bouckenooghe T, Reusens B, Remacle C: Maternal malnutrition programs pancreatic islet mitochondrial dysfunction in the adult offspring. J Nutr Biochem 2011;22:985-994.

58 Nardelli TR, Ribeiro RA, Balbo SL, Vanzela EC, Carneiro EM, Boschero AC, Bonfleur ML: Taurine prevents fat deposition and ameliorates plasma lipid profile in monosodium glutamate-obese rats. Amino Acids 2011;41:901-908.

59 Dalgaard LT: Biochemical and Biophysical Research Communications UCP2 mRNA expression is dependent on glucose metabolism in pancreatic islets. Biochem Biophys Res Commun 2012;417:495-500. 